Corrigendum

\title{
Corrigendum to "Relationship between the Direction of Ophthalmic Artery Blood Flow and Ocular Microcirculation before and after Carotid Artery Stenting"
}

\author{
Masashi Ishii, ${ }^{1}$ Morito Hayashi, ${ }^{1}$ Fumihiko Yagi, ${ }^{2}$ Kenichiro Sato, ${ }^{1}$ Goji Tomita, ${ }^{2}$ \\ and Satoshi Iwabuchi ${ }^{1}$ \\ ${ }^{1}$ Department of Neurosurgery, Toho University Ohashi Medical Center, Tokyo, Japan \\ ${ }^{2}$ Department of Ophthalmology, Toho University Ohashi Medical Center, Tokyo, Japan \\ Correspondence should be addressed to Morito Hayashi; morito@med.toho-u.ac.jp
}

Received 18 September 2017; Accepted 15 October 2017; Published 12 November 2017

Copyright (C) 2017 Masashi Ishii et al. This is an open access article distributed under the Creative Commons Attribution License, which permits unrestricted use, distribution, and reproduction in any medium, provided the original work is properly cited.

In the article titled "Relationship between the Direction of Ophthalmic Artery Blood Flow and Ocular Microcirculation before and after Carotid Artery Stenting" [1], there was an error in Figure 6. For the nonantegrade group $(N=18)$, Figure 6 should show 18 lines, but showed 19 lines because one case from the antegrade group was incorrectly included. This error does not change the statistical results, because the calculation was done correctly on 18 cases. Figure 6 should be corrected as follows:
Additionally, reference 27 was incorrectly quoted. Therefore, the text reading "Haga et al. reported on the utility of LSFG before and after CAS but with a small sample of seven cases [27]" should be changed to "Haga et al. reported on the utility of LSFG before and after CEA but with a small sample of five cases [27]." 

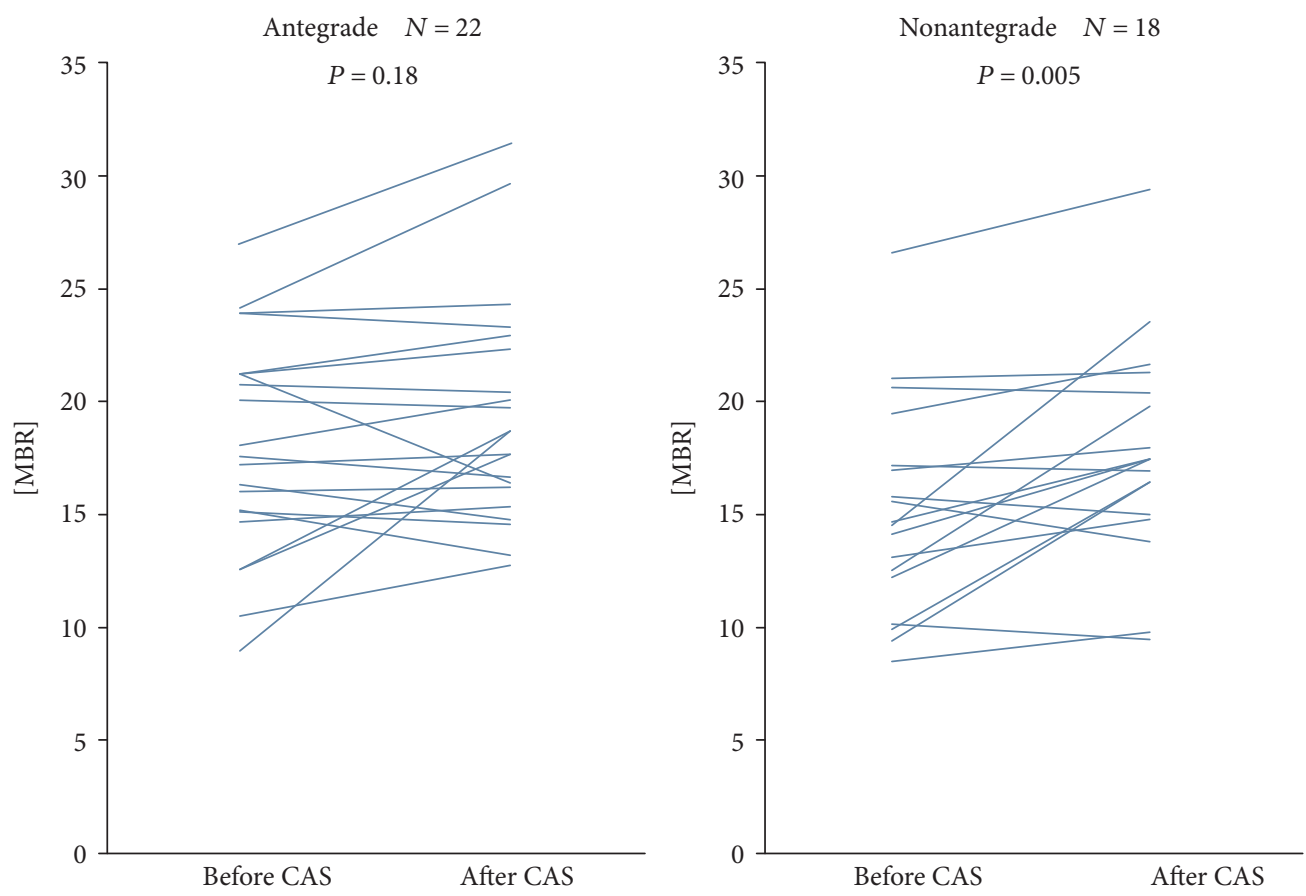

FIGURE 6: Change in the MBR before and after CAS in the antegrade and nonantegrade groups. There was the significant rise in the MBR in the nonantegrade group $(P=0.005)$.

\section{References}

[1] M. Ishii, M. Hayashi, F. Yagi, K. Sato, G. Tomita, and S. Iwabuchi, "Relationship between the direction of ophthalmic artery blood flow and ocular microcirculation before and after carotid artery stenting," Journal of Ophthalmology, vol. 2016, Article ID 2530914, 6 pages, 2016. 


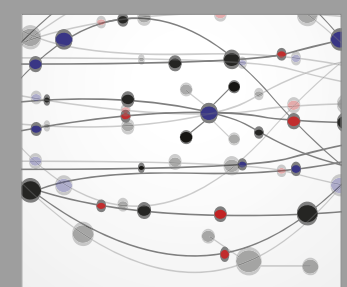

The Scientific World Journal
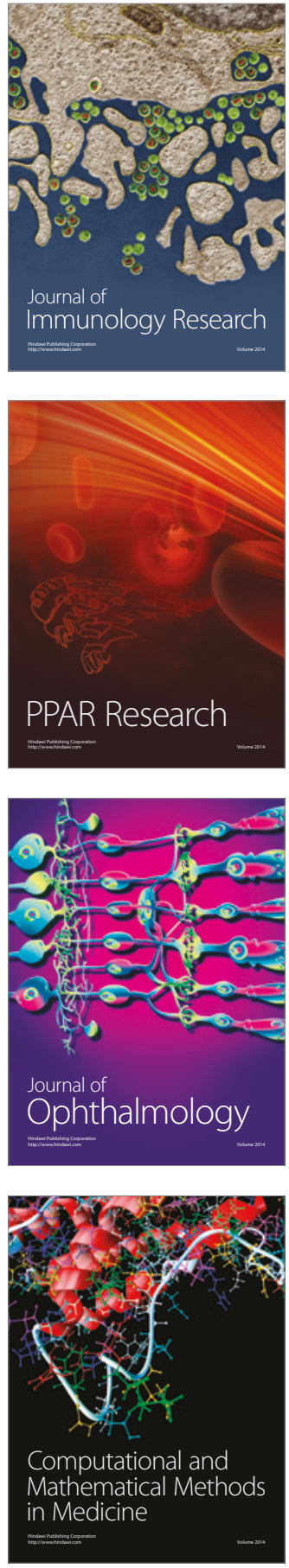

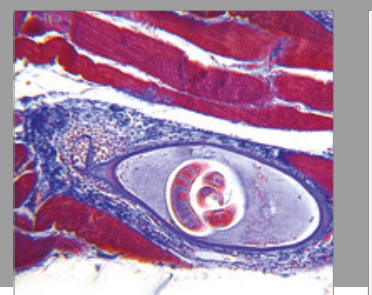

Gastroenterology Research and Practice
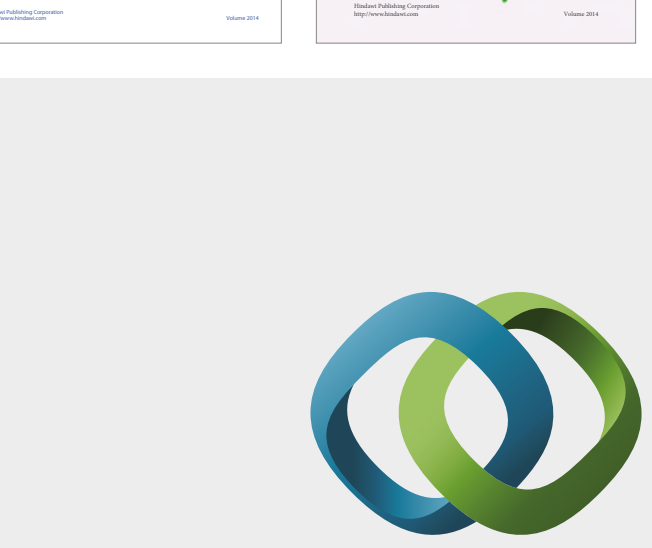

\section{Hindawi}

Submit your manuscripts at

https://www.hindawi.com
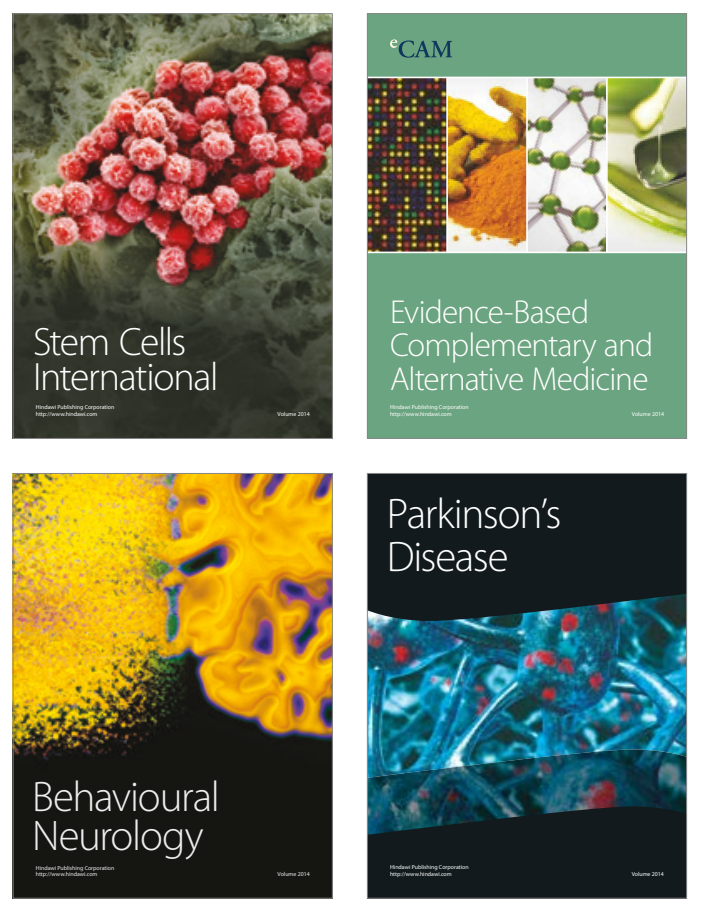
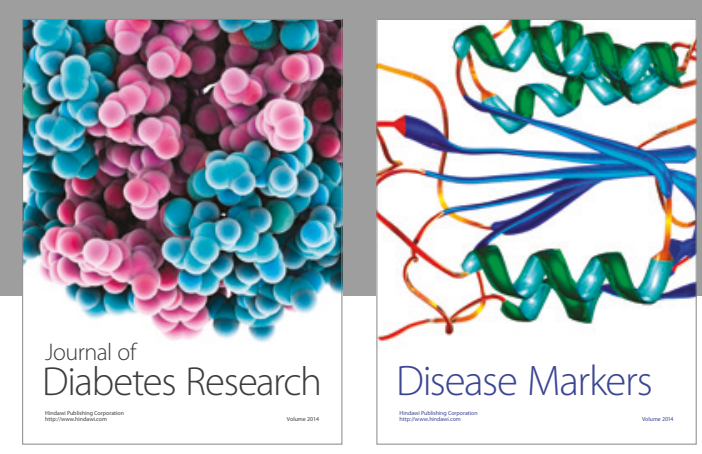

Disease Markers
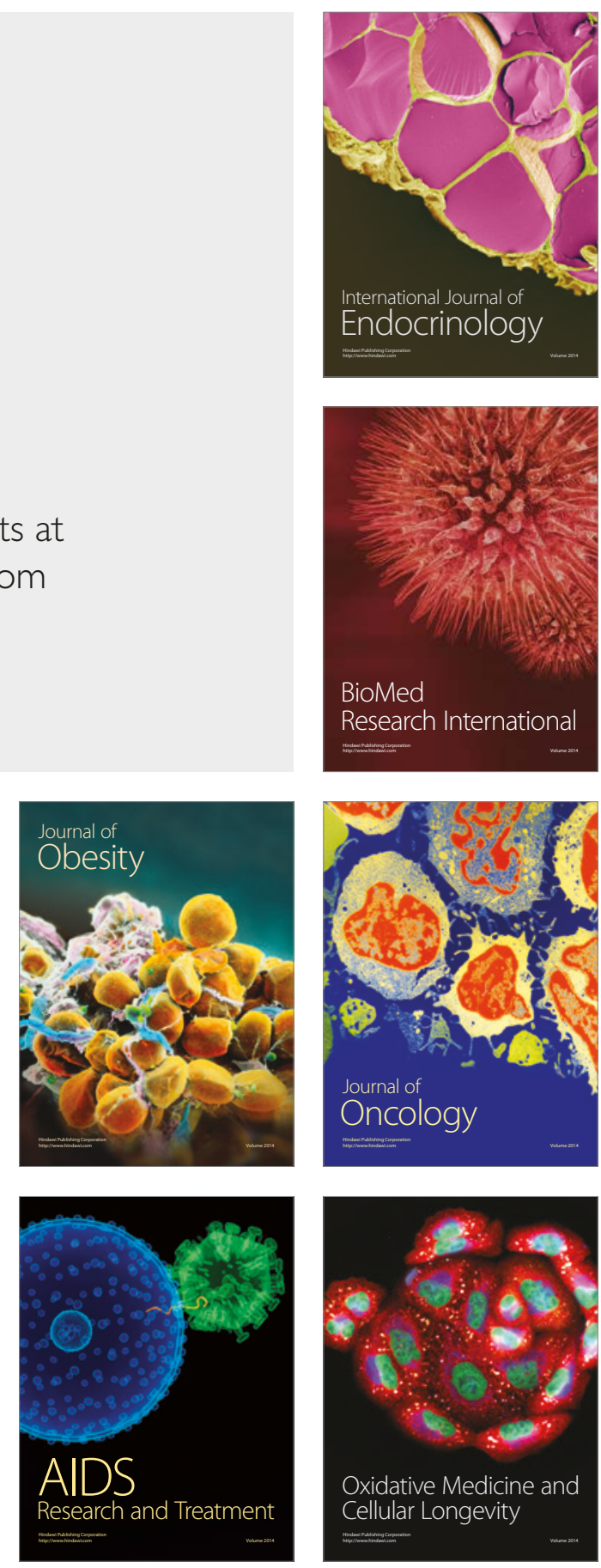